Wright State University

CORE Scholar

Mathematics and Statistics Faculty

Publications

Mathematics and Statistics

$1-1996$

\title{
A Center-Unstable Manifold Theorem for Parametrically Excited Surface Waves
}

Larry Turyn

Wright State University - Main Campus, larry.turyn@wright.edu

Follow this and additional works at: https://corescholar.libraries.wright.edu/math

Part of the Applied Mathematics Commons, Applied Statistics Commons, and the Mathematics Commons

\section{Repository Citation}

Turyn, L. (1996). A Center-Unstable Manifold Theorem for Parametrically Excited Surface Waves. SIAM Journal on Mathematical Analysis, 27 (1), 241-257.

https://corescholar.libraries.wright.edu/math/40

This Article is brought to you for free and open access by the Mathematics and Statistics department at CORE Scholar. It has been accepted for inclusion in Mathematics and Statistics Faculty Publications by an authorized administrator of CORE Scholar. For more information, please contact library-corescholar@wright.edu. 


\title{
A CENTER-UNSTABLE MANIFOLD THEOREM FOR PARAMETRICALLY EXCITED SURFACE WAVES*
}

\author{
LAWRENCE TURYN ${ }^{\dagger}$
}

\begin{abstract}
When fluid in a rectangular tank sits upon a platform which is oscillating with sufficient amplitude, surface waves appear in the "Faraday resonance." Scientists and engineers have done bifurcation analyses which assume that there is a center manifold theory using a finite number of excited spatial modes. We establish such a center manifold theorem for Xiao-Biao Lin's model in which potential flow is assumed but an artificial dissipation term is included in the system of partial differential equations on the free surface. We use interpolation spaces developed by da Prato and Grisvard, establish maximal regularity for a family of evolution operators, and adapt the center manifold theory of Chow, Lin, and Lu.
\end{abstract}

Key words. parametric resonance, center manifold, surface waves, interpolation spaces, maximal regularity

AMS subject classifications. Primary, 76B15, 35G25, 34C45, 34G20; Secondary, 70J40, 35Q35, 47D06, 46B70

1. Introduction and summary. Consider a rectangular tank filled with an incompressible homogeneous fluid to a depth $h$. If the base of the tank is made to oscillate, then what will be the behavior of the fluid? As long ago as 1831, Faraday observed fluid oscillations at one half the frequency of the base, so this phenomenon of parametric excitation is known as Faraday resonance. This and other historical references can be found in Benjamin and Ursell [3] and Miles and Henderson [18].

Lin [15] has established a mathematical formulation for this problem. As long as the amplitude of the excitation is sufficiently small, he has obtained (i) global existence and uniqueness and (ii) an approximation result which justifies a truncation to a finite number of modes. His model assumes potential flow and includes on the free boundary terms for surface tension and artificial viscosity. The latter is to some extent physically meaningful because Lin showed that at high wave numbers his artificial viscosity produces dissipation proportional to the square of the wave number and proportional to the total kinematic energy, i.e., the dissipation is consistent with that produced by kinematic viscosity.

On the other hand, several authors, e.g., Gu and Sethna [14], Holmes [13], and Silber and Knobloch [23], have analyzed bifurcation equations assumed to hold on a finite-dimensional center manifold corresponding to some neutrally stable mode(s). If there is friction in the system, this is only possible if the amplitude of the excitation is not required to be sufficiently small.

The purpose of this paper is to establish the existence of a local center manifold theorem for this problem. We introduce virtually no new mathematical techniques but instead apply a variety of results established by other authors.

Our mathematical formulation is due to Lin [15]. The velocity potential, as a function of the shape of the free surface and the potential on the free surface,

* Received by the editors May 25, 1993; accepted for publication (in revised form) April 15, 1994.

†Department of Mathematics and Statistics, Wright State University, Dayton, OH 45435. This research was partially done while the author was visiting the School of Mathematics, Georgia Institute of Technology, Atlanta, GA 30332. 
is substituted into Bernoulli's equation on the free surface. We use this functional relationship from Lin's paper, albeit in a time-independent version, in Theorem 6.1 below. Our techniques diverge from Lin's in that we then consider the problem as an ordinary differential equation in function spaces of space dependence rather than as an implicit equation in a Hilbert function space of both space and time dependence.

For our approach, we use a variation of constants formula in interpolation spaces, as in da Prato and Grisvard [8], Sinestrari [25], and da Prato and Lunardi [9], which gives "maximal regularity." Unfortunately, we could not use the methods of Henry [12] for our nonlinear problem, although we do mimic his methods for periodic, linear problems. The actual local center manifold theorem that we obtain is an application of Chow, Lin, and Lu [7], or we could have used the result of da Prato and Lunardi [9], which is also in the style of Henry [12, Chap. 6]. The method of Liapunov-Perron obtains an invariant manifold as a graph of a function defined by an integral operator.

The paper is organized in this way: In $\S 2$, the physical problem and a model for it are presented along with a linearization about the flat surface, i.e., undisturbed, solution. In $\S 3$, a semigroup of bounded linear operators is explicitly presented and shown to be analytic. In $\S 4$, a brief description is made of a well-known general method for defining interpolation spaces in which our semigroup has a maximal regularity property. In $\S 5$, we return to linear, time-periodic problems and show in Proposition 5.2 that there is a family of evolution operators which define integral operators with a generalization of the maximal regularity property for autonomous problems. We show that our linearized, time-periodic problem can be analyzed in this way. These generalizations are the only "new" results we have; all the other work in this paper consists of applying the work of other authors to our specific problem or, in the case of Lin's paper, taking a result in its entirety. In $\S 6$, the nonlinearities of our model are found to be nice enough viz. the integral operators of $\S 5$; here a result of Lin is essential. In $\S 7$, we adapt a center-unstable manifold theorem and an exponential attractivity theorem of other authors to our nonlinear, time-periodic problem. In $\S 8$, we mention a result of a sequel in preparation. We have calculated an approximate, local-center manifold for an example involving the $(3,2)$ and $(2,3)$ spatial modes.

In fact, experiments of Simonelli and Gollub [24] provide examples where there are a small number of unstable modes. As an aside, when we examined their experimental data for the onset of instability [24, p. 479, Fig. 4(a)] and used a theoretical result for the damped Mathieu equation from Turyn $[27, \S 1]$, we came to the conclusion that the artificial viscosity term $-\mu \nabla_{\mathbf{x}}^{2} u$ in equation (2.5) of our paper would have $\mu$ about two to three times the kinematic viscosity, 2.948 centipoises, of $n$-butyl alcohol at $20^{\circ} \mathrm{C}$. This suggests that, although this artificial viscosity is consistent with dissipation of kinetic energy, as in Lin $[15, \S 6]$, the mechanism may not be so simple.

Our original intention was to discuss regions more general than rectangular tanks, e.g., cylindrical tanks. We ran into difficulties in $\S 6$ when discussing boundary conditions to be satisfied by the nonlinearities. We hope to understand this better in the future.

2. The physical problem and its linearization. Let $D$ be a bounded open domain in $\mathbb{R}^{2}$, with coordinates $\mathbf{x}$. We will define the smoothness we require of its boundary $\partial D$ later. The moving coordinate $z$ is fixed with respect to the oscillating container, with the positive axis pointing upwards. The free boundary is $S_{F}: z=$ $v(\mathbf{x}, t)$ and we denote

$$
\Omega_{v}=\Omega_{v}(t):=\{(\mathbf{x}, z):-h<z<v(\mathbf{x}, t), \mathbf{x} \in D\}
$$


with outward unit normal $\mathbf{n}$. We assume the velocity field $\mathbf{V}$ is the gradient of a potential $\phi=\phi(\mathbf{x}, z, t)$ which satisfies

$$
\left\{\begin{aligned}
\nabla^{2} \phi & =0 & & \text { in } \Omega_{v} \\
\phi & =u(\mathbf{x}, t) & & \text { on } S_{F}: z=v(\mathbf{x}, t) \\
\frac{\partial \phi}{\partial \mathbf{n}} & =0 & & \text { on } \partial \Omega_{v} \backslash S_{F}
\end{aligned}\right.
$$

where $\nabla=\left(\nabla_{\mathbf{x}}, \frac{\partial}{\partial z}\right)$. The latter boundary condition states that the normal velocity is zero on the sides and base of the container.

On the free boundary the potential $\phi$ and the shape $v$ satisfy the "kinematic" condition

$$
v_{t}=\phi_{z}-\nabla_{\mathbf{x}} \phi \cdot \nabla_{\mathbf{x}} v \quad \text { on } S_{F}
$$

where subscripts denote differentiation, and Bernoulli's equation

$$
\phi_{t}=-\gamma \nabla \cdot \mathbf{n}-(g-\alpha(t)) v-\frac{1}{2}|\nabla \phi|^{2}+\mu \nabla_{\mathbf{x}}^{2} u \quad \text { on } S_{F},
$$

where atmospheric pressure $p_{0} \equiv 0$, density $\rho \equiv 1, \gamma$ is the coefficient of surface tension, $g$ is the acceleration of gravity, the parametric excitation $\alpha(t)$ is the effect of the oscillating base of the container, and we have added an artificial dissipative term proportional to $\nabla_{\mathbf{x}}^{2} u$, as in Lin [15]. Specifically, we have $\alpha(t)=a\left(d^{2} / d t^{2}\right) \cos (\omega t)$, where $a$ is the amplitude of the oscillations of the base of the container.

On the free boundary, $u(\mathbf{x}, t)=\phi(\mathbf{x}, v(\mathbf{x}, t), t)$, so $u_{t}=\phi_{t}+\phi_{z} v_{t}$, etc. Denote $w(\mathbf{x}, t):=\phi_{z}(\mathbf{x}, v(\mathbf{x}, t), t)$, so that $w=N(v) u$ is a linear operator on $u$ which depends nonlinearly on $v$. In Theorem 6.1, we will borrow from Lin [15] a result on the smooth dependence of $w$ on $u$ and $v$, in suitable function spaces of spatial dependence.

Denote by [ ] the operation of taking the mean over the domain $D$, i.e., $D[f]=$ $\int_{D} f$. First, we modify the model so as to have the property $[u]=0$ preserved in time; the property $[v]=0$ is automatically preserved. Second, we replace the nonlinear surface tension term $-\gamma \nabla \cdot \mathbf{n}$, where $\mathbf{n}=\left(-\nabla_{\mathbf{x}} v, 1\right) / \sqrt{1+\left|\nabla_{\mathbf{x}} v\right|^{2}}$ on the free surface, by $\gamma \nabla_{\mathbf{x}}^{2} v$, its linearization about the undisturbed solution $v \equiv 0$. In Lin's paper one finds both modifications; however, in his paper the second modification is done purely for convenience. In fact, because his spaces $K(r, s)$ satisfy $K(r, 0) \subseteq K(r, 2)$ for $r>2$, he can treat the nonlinear surface tension term as easily as its linearization. In our formulation, it seems we must make this second modification, otherwise the spaces $E$ and $F$, described in $\S 4$, will not be suitable in $\S 6$ for $\mathbf{f}: F \rightarrow E$. It is not clear if this second modification is acceptable viz. physical experiments, for small oscillations.

In terms of $v, u, w$ these modifications of (2.4) and (2.5) yield

$$
\left\{\begin{array}{l}
v_{t}=w+M_{1}(v, u) \\
u_{t}=\gamma \nabla_{\mathbf{x}}^{2} v+\mu \nabla_{\mathbf{x}}^{2} u-(g-\alpha(t)) v+M_{2}(v, u)-\left[M_{2}(v, u)\right]
\end{array}\right.
$$

where

$$
\begin{gathered}
M_{1}(v, u)=w\left|\nabla_{\mathbf{x}} v\right|^{2}-\nabla_{\mathbf{x}} u \cdot \nabla_{\mathbf{x}} v, \\
M_{2}(v, u)=-\frac{1}{2}\left|\nabla_{\mathbf{x}} u\right|^{2}+\frac{1}{2} w^{2}\left(1+\left|\nabla_{\mathbf{x}} v\right|^{2}\right) .
\end{gathered}
$$

We take as boundary conditions $\frac{\partial u}{\partial \mathbf{n}}=0$ on $\partial D$, which follows from (2.3), and $\frac{\partial v}{\partial \mathbf{n}}=0$ on $\partial D$. The latter is somewhat controversial; in fact, one could argue that 
instead of an artificial dissipation term $\mu \nabla_{\mathbf{x}}^{2}$ in equation (2.5), one should introduce damping in the boundary conditions. This has been brought to my attention by $\mathrm{M}$. Silber. Recently, Simonelli and Gollub [24] have described experiments in which the free surface is at right-angle contact with the sidewalls of a rectangular container, and this is consistent with $\frac{\partial v}{\partial \mathbf{n}}=0$ on $\partial D$. Douady [10] has described experiments in which the free surface is pinned at the boundary, i.e., $v=0$ on $\partial D$, by the use of felt on the walls of the container. It appears that this boundary condition cannot be accommodated by our abstract framework.

To linearize equations (2.6) and (2.7), we need only replace $w$ by $N(0) u$ in equation (2.6) and replace $M_{1}$ and $M_{2}$ by 0 . To this end, let $A:=-\Delta_{N}$ denote the Neumann Laplacian on the complex Hilbert space $\tilde{L}^{2}(D)=\left\{u \in L^{2}(D): \int_{D} u=0\right\}$, with domain $\mathcal{D}(A)=\left\{u \in \tilde{L}^{2}(D): A u \in \tilde{L}^{2}(D), \frac{\partial u}{\partial \mathbf{n}}=0\right.$ on $\left.\partial D\right\}$. Throughout, assume that $D \subseteq \mathbb{R}^{2}$ is bounded and open and has the uniform $C^{m}$ regularity property, as in Adams [1, p. 67], with $m$ as large as needed later. It is well known that $A: \mathcal{D}(A) \subseteq$ $\tilde{L}^{2}(D) \rightarrow \tilde{L}^{2}(D)$ is a strictly positive definite, self-adjoint linear operator with compact inverse. Let $\left\{\kappa_{n}^{2}\right\}$ be the eigenvalues of $A$ and $\psi_{n}=\psi_{n}(\mathbf{x})$ be the corresponding orthonormalized eigenfunctions; without loss of generality, $\kappa_{1}^{2} \leq \kappa_{2}^{2} \leq \cdots$. As in Henry [12] or Pazy [21], one can define the fractional powers $A^{\beta}$, which in this situation are closed linear operators with domain $X^{\beta}:=\left\{u \in \tilde{L}^{2}(D): \sum_{n=1}^{\infty} \kappa_{n}^{4 \beta}\left|\left(u, \psi_{n}\right)_{L^{2}}\right|^{2}<\infty\right\}$. From now on we will write $\sum_{n}$ instead of $\sum_{n=1}^{\infty}$ and denote $u_{n}=\left(u, \psi_{n}\right)_{L^{2}(D)} . X^{\beta}$ is a Hilbert space when given the inner product $(u, v)=\sum_{n} \kappa_{n}^{4 \beta} u_{n} \bar{v}_{n}$.

From inspection of $(2.1)-(2.3)$, Lin $[15, \S 4]$ obtained the explicit result that

$$
N(0) u=\sum_{n} \kappa_{n} \tan h\left(\kappa_{n} h\right) u_{n} \psi_{n} \quad \text { for } u=\sum_{n} u_{n} \psi_{n}
$$

It follows that for any $\beta, N(0): X^{\beta} \rightarrow X^{\beta-(1 / 2)}$ is a bounded linear operator. In fact $N(0)$ is close to being $A^{1 / 2}=\left(-\Delta_{N}\right)^{1 / 2}$. To be precise, $N(0)=A^{1 / 2}+A_{1}$, where

$$
A_{1} u=\sum_{n} \kappa_{n}\left(\tan h\left(\kappa_{n} h\right)-1\right) u_{n} \psi_{n}
$$

Since $0<\kappa_{n} \rightarrow \infty$ as $n \rightarrow \infty, A_{1}: X^{\beta} \rightarrow X^{\beta}$ is a bounded linear operator. Thus, the linearization of (2.6)-(2.7) about $u=v=0$ is

$$
\frac{d}{d t}\left(\begin{array}{l}
v \\
u
\end{array}\right)=B\left(\begin{array}{l}
v \\
u
\end{array}\right)+B_{1}(t)\left(\begin{array}{l}
v \\
u
\end{array}\right)
$$

where

$$
B=\left(\begin{array}{cc}
0 & A^{1 / 2} \\
-\gamma A & -\mu A
\end{array}\right), \quad B_{1}(t)=\left(\begin{array}{cc}
0 & A_{1} \\
-(g-\alpha(t)) I & 0
\end{array}\right)
$$

Our plan for the rest of the paper is, first, to analyze the semigroup of linear operators $e^{t B}$ on spaces which will be suitable for subsequent analysis; second, to express the solution of (2.8) in terms of a family of evolution operators; third, to establish a local existence and uniqueness theorem for the nonlinear problem (2.6)(2.7); and finally, to establish a local center manifold theorem for (2.6)-(2.7).

3. The semigroup $e^{t B}$. First, we analyze the semigroup $e^{t B}$ on the Hilbert space $\mathbf{X}:=X^{\beta} \times X^{\beta-(1 / 2)}$, for all $\beta \geq \frac{1}{2}$. We will be more specific about $\beta$ when 
discussing the nonlinear problem in $\S 6$. Other choices of $\mathbf{X}$ are possible but less convenient for our subsequent analysis of $\mathcal{D}(B), \mathcal{D}\left(B^{2}\right)$, and the nonlinear problem. Choose $\mathcal{D}(B)=X^{\beta+(1 / 2)} \times X^{\beta+(1 / 2)}$, which is itself a Hilbert space. From (2.9) $B: \mathcal{D}(B) \subset \mathbf{X} \rightarrow \mathbf{X}$ is a closed, densely defined linear operator. In addition, $B$ has a compact resolvent, as one can see by explicit calculation of $(\lambda I-B)^{-1}$ as the operator norm limit of natural finite-rank operators. The spectrum of $B$ consists of those $\lambda \in \mathbb{C}$ which are eigenvalues and thus solve, for some $n \geq 1$,

$$
\lambda^{2}+\mu \kappa_{n}^{2} \lambda+\gamma \kappa_{n}^{3}=0 .
$$

For future reference, we note that $0 \notin \sigma(B)$. Let

$$
\lambda_{n}^{ \pm}:=\frac{1}{2} \kappa_{n}^{2}\left(-\mu \pm \delta_{n}\right), \delta_{n}:=\left[\mu^{2}-4 \gamma \kappa_{n}^{-1}\right]^{1 / 2} .
$$

The corresponding eigenfunctions of $B$ are

$$
\left(\begin{array}{c}
\mu \pm \delta_{n} \\
-2 \gamma
\end{array}\right) \psi_{n}
$$

For convenience, assume $\delta_{n} \neq 0$ for all $n$. If $\delta_{n}=0$ for a single $n$, the forms of $e^{t B}$ and $B e^{t B}$ would be altered, but the results, (3.3) and following, would not be affected. By diagonalizing $B$ in each of the subspaces spanned by

$$
\left\{\left(\begin{array}{c}
\psi_{n} \\
0
\end{array}\right),\left(\begin{array}{c}
0 \\
\psi_{n}
\end{array}\right)\right\}
$$

after much calculation one finds that

$$
e^{t B}\left(\begin{array}{c}
v \\
u
\end{array}\right)=\sum_{n} \delta_{n}^{-1}\left(e^{t \lambda_{n}^{+}} C_{n}^{+}+e^{t \lambda_{n}^{-}} C_{n}^{-}\right)\left(\begin{array}{c}
v_{n} \\
u_{n}
\end{array}\right) \psi_{n},
$$

where $v_{n}:=\left(v, \psi_{n}\right)=\int_{D} v \psi_{n}, u_{n}:=\left(u, \psi_{n}\right)$, and

$$
C_{n}^{+}:=\left(\begin{array}{cc}
\mu\left(\nu_{n}^{+}\right)^{-1} & \kappa_{n}^{-1} \\
-\gamma & -\frac{\gamma}{\mu} \nu_{n}^{+} \kappa_{n}^{-1}
\end{array}\right), \quad C_{n}^{-}:=\left(\begin{array}{cc}
-\frac{\gamma}{\mu}\left(\nu_{n}^{-}\right)^{-1} \kappa_{n}^{-1} & -\kappa_{n}^{-1} \\
\gamma & \mu \nu_{n}^{-}
\end{array}\right),
$$

and $\nu_{n}^{+}:=-\mu \lambda_{n}^{+} /\left(\gamma \kappa_{n}\right), \nu_{n}^{-}:=-\lambda_{n}^{-} /\left(\mu \kappa_{n}^{2}\right)$. One notes that because $\kappa_{n} \rightarrow \infty$ as $n \rightarrow \infty, \lim _{n \rightarrow \infty} \nu_{n}^{ \pm}=1$ and, in fact, $1 \leq \nu_{n}^{+} \leq 2$ and $0<\nu_{n}^{-} \leq 1$ for all $n$, so (3.1) and (3.2) will be convenient for subsequent analysis.

The norm on $\mathbf{X}=X^{\beta} \times X^{\beta-(1 / 2)}$ is given by

$$
\left\|\left(\begin{array}{c}
v \\
u
\end{array}\right)\right\|_{\mathcal{X}}^{2}=\sum_{n}\left(\left(\kappa_{n}^{2 \beta}\left|v_{n}\right|\right)^{2}+\left(\kappa_{n}^{2 \beta-1}\left|u_{n}\right|\right)^{2}\right),
$$

so one sees immediately that (3.1) defines a $C^{0}$, i.e., strongly continuous, semigroup $e^{t B}$ of bounded linear operators on $\mathbf{X}$. We note that, because $\kappa_{n} \rightarrow \infty$ as $n \rightarrow \infty, \sigma(B)$ consists of two infinite sequences of real numbers tending to $-\infty$ and possibly a finite number of complex conjugate pairs of eigenvalues $\left\{\lambda_{n}^{ \pm}\right\}_{1 \leq n \leq n_{0}}$, all with $\operatorname{Re} \lambda_{n}^{ \pm}<0$, so $\left\{e^{t B}\right\}_{t \geq 0}$ is uniformly bounded. We calculate that

$$
B e^{t B}\left(\begin{array}{c}
v \\
u
\end{array}\right)=\sum_{n} \delta_{n}^{-1}\left(\lambda_{n}^{+} e^{t \lambda_{n}^{+}} C_{n}^{+}+\lambda_{n}^{-} e^{t \lambda_{n}^{-}} C_{n}^{-}\right)\left(\begin{array}{c}
v_{n} \\
u_{n}
\end{array}\right) \psi_{n},
$$


so there exists a constant $M$ such that

$$
\left\|t B e^{t B}\right\| \leq M \text { for } t \geq 0
$$

In addition, $e^{t B}$ is differentiable for $t>0$ and $0 \notin \sigma(B)$. It follows from Pazy [21, Thm. 2.5.2] that (i) $\left\{e^{t B}\right\}_{t \geq 0}$ is an analytic semigroup of bounded linear operators on $\mathbf{X}$ and (ii) $-B$ is a sectorial operator, in the sense of Henry [12].

4. Interpolation spaces for autonomous problems. Throughout this section, $\theta$ is a fixed real number, $0<\theta<1$.

In the approach of Henry [12] to nonlinear problems and invariant manifolds, for a problem $\frac{d w}{d t}=B w+f(w), w \in \mathbf{X}$, if $-B$ is a sectorial operator generating an analytic semigroup $e^{t B}$, then one defines $\mathbf{X}^{\rho}=\mathcal{D}\left((-B)^{\rho}\right)$ and hypothesizes that $f: \mathbf{X}^{\rho} \rightarrow \mathbf{X}$ for some $0 \leq \rho<1$. Unfortunately, the nonlinear problem of ours has a specific nonlinearity which does not allow this approach. From Lin [15], we know that our nonlinearity takes $H^{r}(D) \times H^{r}(D)$ into $H^{r-1}(D) \times H^{r-1}(D)$ as long as $r>2$; see Theorem 6.1 below. It seems that if $\mathbf{X}=X^{\beta_{1}} \times X^{\beta_{2}}$, whenever we choose $\beta_{1}, \beta_{2}>\frac{1}{2}$, in order to have $H^{r-1}(D) \times H^{r-1}(D) \subseteq \mathbf{X}$, it follows that to have $\mathbf{X}^{\rho} \subseteq H^{r}(D) \times H^{r}(D)$, one must take $\rho=1$. For example, the calculations of Chen and Triggiani [5] and Rodriguez-Bernal [22] confirm this for many choices of $\beta_{1}, \beta_{2}$; the details are omitted except to note that one can introduce $x=v$ and $y=A^{1 / 2} u$ and define operators

$$
\mathcal{A}=A^{3 / 2} \quad \text { and } \quad B=\left(\begin{array}{cc}
0 & I \\
-\gamma \mathcal{A} & -\mu \mathcal{A}^{2 / 3}
\end{array}\right) .
$$

In addition, the form of $e^{t B}$ leads one to suspect that, for all choices of $\beta_{1}$ and $\beta_{2}$, one must take $\rho=1$.

We can use another approach using interpolation spaces, as in daPrato and Grisvard [8] and many others, including Butzer and Berens [4], Sinestrari [25], daPrato and Lunardi [9], and Angenent [2]. We will denote $E:=D_{B}(\theta)$, to be defined below, and $F:=D_{B}(\theta+1)=\left\{w \in \mathcal{D}(B): B w \in D_{B}(\theta)\right\}$, and we will show in later sections, first, that the linear periodic problem (2.8) has some nice properties with regard to these spaces $E$ and $F$ and, second, that the nonlinearities in problem (2.6)-(2.7) take $F$ into $E$ nicely. The outstanding property of the spaces $E$ and $F$ is "maximal regularity," which has been put to good use by daPrato and Lunardi [9] to establish a center manifold theorem for autonomous problems.

We follow the exposition by Angenent [2] of the daPrato and Grisvard construction [8]. Suppose $E_{0}$ and $E_{1}$ are real Banach spaces with $E_{1}$ densely included in $E_{0}$ and $B: E_{1} \rightarrow E_{0}$ is bounded and linear. Considered as an unbounded operator on $E_{0}$, we assume that $B$ generates an analytic semigroup $e^{t B}$. Throughout this section, $\theta$ is a fixed real number, $0<\theta<1$. We define $E_{\theta}$ to be a space of traces, i.e., initial values, of a certain class of functions

$$
Y_{\theta}:=\left\{y \in C\left((0,1] ; E_{1}\right) \cap C^{1}\left((0,1] ; E_{0}: \lim _{t \downarrow 0} t^{1-\theta}\left(\left\|y^{\prime}(t)\right\|_{E_{0}}+\|y(t)\|_{E_{1}}\right)=0\right\}\right.
$$

with norm

$$
\|y\|_{\theta}:=\sup _{0<t \leq 1} t^{1-\theta}\left(\left\|y^{\prime}(t)\right\|_{E_{0}}+\|y(t)\|_{E_{1}}\right)
$$

Specifically,

$$
E_{\theta}:=\left\{w: w=y(0) \text { for some } y \in Y_{\theta}\right\}
$$


with norm

$$
\|w\|_{\theta}=\inf \left\{\|y\|_{\theta}: y \in Y_{\theta}, y(0)=w\right\} .
$$

It is known that $E_{\theta}$ is a Banach space with this norm and that $E_{\theta}=D_{B}(\theta)$ as defined in daPrato and Lunardi [9]. Similarly, between the spaces $E_{1}$ and $E_{2}:=$ $\mathcal{D}_{E_{1}}(B)=\left\{w \in E_{1}: B w \in E_{1}\right\}$, one can define $E_{1+\theta}$. If $E_{0}=\mathbf{X}$ and $E_{1}=\mathcal{D}(B)$, then $E_{2}=\mathcal{D}\left(B^{2}\right), E_{1+\theta}=D_{B}(\theta+1)$, and $\mathcal{D}\left(B^{2}\right) \subseteq D_{B}(\theta+1) \subseteq \mathcal{D}(B) \subseteq D_{B}(\theta) \subseteq \mathbf{X}$, as in daPrato and Grisvard [8] and Sinestrari [25].

These interpolation spaces are defined by using continuous functions, so in the general theory of interpolation, $E_{\theta} \subseteq\left(E_{1}, E_{0}\right)_{\theta, \infty}$. The interpolation in Lions and Magenes [16] uses $L^{2}$ functions and can be notated $(,)_{1-\theta, 2}$.

One should note that, in our problem, $E_{0}=\mathbf{X}$ and $E_{1}=\mathcal{D}(B)$ are spaces of functions of $\mathbf{x} \in D$, as are $D_{B}(\theta)$ and $D_{B}(\theta+1)$; the time variable appears only in the definition of these spaces as initial values of functions of a time variable.

Because $B$ generates an analytic semigroup $e^{t B}$, there exists $\omega, M$ such that $\left\|e^{t B}\right\|_{L(\mathbf{X})} \leq M e^{\omega t}$, where $L(\mathbf{X})$ denotes the space of bounded linear operators from $\mathbf{X}$ to $\mathbf{X}$. It is known that

$$
\left\|e^{t B}\right\|_{L(Z)} \leq M e^{\omega t}
$$

and that $-B$ is sectorial on $Z$, for any choice of $Z$ being $\mathbf{X}, \mathcal{D}(B), D_{B}(\theta)$, or $D_{B}(\theta+1)$, although it might be necessary to increase $M$.

In our specific problem, the spectrum of $B$ consists of $\left\{\lambda_{n}^{ \pm}\right\}_{n \geq 1}$, where

$$
\lambda_{n}^{ \pm}=-\frac{1}{2} \kappa_{n}^{2}\left(\mu \mp \sqrt{\mu^{2}-4 \gamma \kappa_{n}^{-1}}\right.
$$

Because $0<\kappa_{1} \leq \kappa_{2} \leq \cdots$, there exists $\omega<0$ such that $\operatorname{Re} \sigma(B)<\omega$ and there exists $M \geq 1$ such that (4.1) holds with that $\omega<0$.

In our problem, we take $E_{0}=\mathbf{X}=X^{\beta} \times X^{\beta-(1 / 2)}$ and $E_{1}=\mathcal{D}(B)=X^{\beta+(1 / 2)} \times$ $X^{\beta+(1 / 2)}$. One can calculate that $E_{2}=\mathcal{D}\left(B^{2}\right)=\{w \in \mathcal{D}(B): B w \in \mathcal{D}(B)\}=$ $X^{\beta+1} \times X^{\beta+1} \cap\left\{(v, u): \gamma v+\mu u \in X^{\beta+(3 / 2)}\right\}$. The latter expresses an "interaction condition." One can observe that interpolation commutes with the operation of taking direct products, so that

$$
\begin{aligned}
E_{\theta} & =\left(X^{\beta+1 / 2} \times X^{\beta+1 / 2}, X^{\beta} \times X^{\beta-1 / 2}\right)_{\theta, \infty} \\
& =\left(X^{\beta+1 / 2}, X^{\beta}\right)_{\theta, \infty}+\left(X^{\beta+1 / 2}, X^{\beta-1 / 2}\right)_{\theta, \infty} .
\end{aligned}
$$

We recall that $X^{\beta}=\mathcal{D}\left(A^{\beta}\right)$ where $A=-\Delta_{N}$ on $\tilde{L}^{2}(D)$.

It is known from work of Grisvard [11] that, regarding $0<\beta<1$,

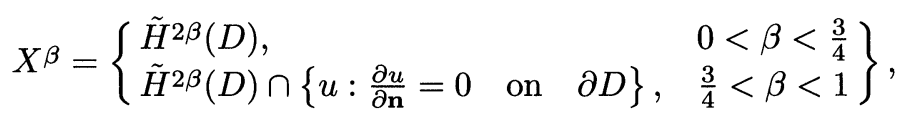

where $H^{s}(D)$ is the Sobolev space $W^{s, 2}(D)$ and, as before, a indicates "mean value zero." Put loosely, in $X^{\beta}$ the boundary condition $\frac{\partial u}{\partial \mathbf{n}}=0$ applies if it makes sense, i.e., if $\left.\nabla_{\mathbf{x}} u\right|_{\partial D}$ makes sense as a trace of a distribution on $\partial D$. For $1<\beta<2$,

$$
\begin{aligned}
X^{\beta} & =\left\{u \in X^{\beta-1}: \Delta_{N} u \in X^{\beta-1}\right\} \\
& =\left\{\begin{array}{ll}
\tilde{H}^{2 \beta} \cap\left\{u: \frac{\partial u}{\partial \mathbf{n}}=0 \text { on } \partial D\right\}, & 1<\beta<\frac{7}{4} \\
\tilde{H}^{2 \beta} \cap\left\{u: \frac{\partial u}{\partial \mathbf{n}}=\frac{\partial \Delta u}{\partial n}=0 \text { on } \partial D\right\}, & \frac{7}{4}<\beta<2
\end{array}\right\} .
\end{aligned}
$$


It is known from daPrato and Grisvard $[8, \S 6.1]$ that

$$
\left(X^{\beta+1 / 2}, X^{\beta}\right)_{\theta, \infty} \subseteq\left\{u \in X^{\beta}: A^{\beta} u \in h_{2}^{\theta}\right\}
$$

and

$$
\left(X^{\beta+1 / 2}, X^{\beta-1 / 2}\right)_{\theta, \infty} \subseteq\left\{u \in X^{\beta-1 / 2}: A^{\beta-1 / 2} u \in h_{2}^{2 \theta}\right\} .
$$

Here $h_{2}^{s}(D)$ stands for a Nikolsk'ii space, as in Nikolsk'ii [19], [20], Slobodeckii [26], and Adams [1]. These spaces are actually a subclass of the Besov spaces. The only property of these spaces we will use are the continuous imbeddings

$$
H^{s}(D) \hookrightarrow h^{s}(D) \hookrightarrow H^{s-\varepsilon}(D)
$$

for any $\varepsilon>0$. We will use the property that interpolation preserves bounded linear operators in order to avoid the need for detailed examination of the Nikolsk'ii spaces.

For the moment, we leave our specific operator $B$ and specific space $\mathbf{X}$ and return now to the general situation. We denote $E=E_{\theta}=D_{B}(\theta)$ and $F=E_{1+\theta}=D_{B}(\theta+1)$. The maximal regularity property mentioned above is as follows:

$$
\text { for all } f \in C([0, \tau) ; E), \quad \int_{0}^{\cdot} e^{(\cdot-s) B} f(s) d s \in C([0, \tau] ; F) \text {. }
$$

Proofs can be found in daPrato and Grisvard [8], Sinestrari [25], and Angenent [2], among others. We apply results of this sort to our specific operator $B$ and space $\mathbf{X}$ in Propositions 4.1 and 4.2 below.

Many proofs of existence of invariant manifolds explicitly or implicitly involve weighted spaces; see, for example, Chow, Lin, and $\mathrm{Lu}$ [7] and van Gils and Vanderbauwhede [28]. For a Banach space $Z, \sigma \in \mathbb{R}, \eta \in \mathbb{R}$, define

$$
C_{\eta}((-\infty, \sigma] ; Z)=\left\{f: f \text { continuous on }(-\infty, \sigma] \text { and }\|f\|_{\eta, \sigma,-, Z}<\infty\right\} \text {, }
$$

where its norm is

$$
\|f\|_{\eta, \sigma,-, Z}=\sup _{t \leq \sigma} e^{-\eta t}|f(t)|_{Z}
$$

Similarly define

$$
C_{\eta}([\sigma, \infty) ; Z)=\left\{f: f \text { continuous on }[\sigma, \infty) \text { and }\|f\|_{\eta, \sigma,+, Z}<\infty\right\}
$$

where

$$
\|f\|_{\eta, \sigma,+, Z}=\sup _{t \geq \sigma} e^{\eta t}|f(t)|_{Z}
$$

Recall that in our problem $\left\|e^{t B}\right\|_{L(Z)} \leq M e^{\omega t}$, for some $\omega<0$ and $M \geq 1$, from (4.1), where $Z$ is any of $\mathbf{X}, \mathcal{D}(B), D_{B}(\theta), D_{B}(\theta+1)$. Here, $\theta$ is a fixed real number with $0<\theta<1$. We apply two results of daPrato and Lunardi $[9$, pp. 118-120] to our specific $B$ and $\mathbf{X}$ to get the following propositions.

Proposition 4.1. With $B$ as in (2.9), $\mathbf{X}=X^{\beta} \times X^{\beta-(1 / 2)}, \mathcal{D}(B)=X^{\beta+(1 / 2)} \times$ $X^{\beta+(1 / 2)}$, and $M, \omega$ as in (4.1), choose any $\eta$ such that $\omega+\eta<0$. Then there exists $K_{+}=K_{+}(\eta, \omega, \sigma, \theta)$ such that $f(\cdot) \mapsto \int_{\sigma}^{\cdot} e^{(\cdot-s) B} f(s) d s$ defines a bounded linear operator $\mathcal{L}_{+}: C_{\eta}([\sigma, \infty) ; E) \rightarrow C_{\eta}([\sigma, \infty) ; F)$ with $\left\|\mathcal{L}_{+}\right\| \leq K_{+}$, where $E=D_{B}(\theta), F=$ $D_{B}(\theta+1)$. 
Proposition 4.2. With $B, M$ and $\omega$, as in Proposition 4.1, choose any $\eta$ such that $\omega-\eta<0$. Then there exists $K_{-}=K_{-}(\eta, \omega, \sigma, \theta)$ such that

$$
f(\cdot) \mapsto \int_{-\infty}^{\cdot} e^{(\cdot-s) B} f(s) d s
$$

defines a bounded linear operator $\mathcal{L}_{-}: C_{\eta}((-\infty, \sigma] ; E) \rightarrow C_{\eta}((-\infty, \sigma] ; F)$ with $\left\|\mathcal{L}_{-}\right\| \leq$ $K_{-}$, where $E=D_{B}(\theta)$ and $F=D_{B}(\theta+1)$.

These two propositions express a maximal regularity property useful for the proof of the existence of an invariant manifold for an autonomous problem. In the next section, these two propositions will imply similar properties for a periodic problem.

5. A family of evolution operators for equation (2.8). Given two normed linear spaces $Z_{1}$ and $Z_{2}, L\left(Z_{1}, Z_{2}\right)$ denotes the space of bounded linear operators with the operator norm.

In this section, we establish a new result for periodic problems considered in the framework of interpolation spaces.

Definition. A family of evolution operators $\{\Phi(t, s)\}$ consists of bounded linear operators on a space $Z$ for $t \geq s$ satisfying $\Phi(s, s)=I, \Phi\left(t_{1}, s\right) \Phi\left(s, t_{2}\right)=\Phi\left(t_{1}, t_{2}\right)$ for all $t_{1} \geq s \geq t_{2}$ in $\mathbb{R}$, and $\{\Phi(t, s): t \geq s\}$ is strongly continuous in $(t, s)$ with values in $L(Z)$.

Assume $-B$ is sectorial on a Banach space and $t \mapsto B_{1}(t): \mathbb{R} \rightarrow L\left(E^{\rho}, E\right)$ is Hölder-continuous with exponent $<1$. The work of Henry [12, §7.1] shows that the solutions of

$$
\left\{\begin{array}{l}
\frac{d w}{d t}=B w+B_{1}(t) w \\
w(s)=w_{0} \in E
\end{array}\right\}
$$

are given by $w(t)=\Phi(t, s) w_{0}$, where $\{\Phi(t, s)\}$ is a family of evolution operators which satisfies

$$
\Phi(t, s)=e^{(t-s) B}+\int_{s}^{t} e^{(t-\tau) B} B_{1}(\tau) \Phi(\tau, s) d \tau
$$

for $t \geq s$. Moreover, if $B_{1}(\cdot)$ is periodic with period $T$, then $\Phi(t+T, s+T)=\Phi(t, s)$ for $t \geq s$.

In our particular problem (2.8), we can take $\rho=0$, i.e., $E^{\rho}=E$.

For the periodic problem (5.1), one can define the period map $U(t)=\Phi(t+T, t)$. In our problem, $B$ has compact resolvent on $\mathbf{X}$. The same argument, approximation in the uniform operator norm by a sequence of finite rank operators, shows that $B$ has compact resolvent on $\mathcal{D}(B)$. By interpolation, we see that $B$ has compact resolvent on $E:=D_{B}(\theta)$, where we fix any $\theta \in(0,1)$. From Henry [12, $\left.\S 7.2\right]$, the nonzero eigenvalues of $U(t)$ are independent of time $t$ and constitute all of the spectrum of $U(t)$ with the exception of 0 , which is in the continuous spectrum of $U(t)$ since it is a compact operator.

Proposition 5.1 (Henry [12, Thm. 7.2.3 et seq.]). If all of the characteristic multipliers of the problem (2.8), i.e ., problem (5.1) in our specific case, are of modulus less than 1 and bounded away from 1 , then there exist $\tilde{M} \geq 1$ and $\tilde{\omega}<0$ such that

$$
\|\Phi(t, s)\|_{L\left(E, E^{\rho}\right)} \leq \tilde{M}(t-s)^{-\rho} e^{\tilde{\omega}(t-s)}, \quad \text { for } t>s .
$$

Proof. It suffices to show that $t \mapsto B_{1}(t):[0, T] \rightarrow L\left(E^{\rho}, E\right)$ is Hölder continuous. Looking at (2.9) we see that $t \mapsto B_{1}(t):[0, T] \rightarrow L(\mathcal{X})$ and $t \mapsto B_{1}(t)$ : 
$[0, T] \rightarrow L(\mathcal{D}(B))$ are Hölder continuous, so the desired result follows by interpolation of bounded linear operators.

Proposition 5.2. Assume that $B_{1}(\cdot)$ is periodic with period $T, B_{1}(\cdot):[0, T] \rightarrow$ $L\left(E^{\rho}, E\right)$ is Hölder continuous, and (5.3) holds, apart from any assumption as to the sign of $\tilde{\omega}$. If $\eta$ is chosen so that $\tilde{\omega}+\eta<0$ and $\tilde{\omega}-\eta<0$, then, for all $\sigma \in \mathbb{R}$, the map

$$
f(\cdot) \mapsto \int_{-\infty}^{\cdot} \Phi(\cdot, s) f(s) d s
$$

defines a bounded linear operator

$$
\mathcal{L}_{-}: C_{\eta}((-\infty, \sigma] ; E) \rightarrow C_{\eta}((-\infty, \sigma] ; F)
$$

and the map

$$
f(\cdot) \mapsto \int_{\sigma}^{\cdot} \Phi(\cdot, s) f(s) d s
$$

defines a bounded linear operator

$$
\mathcal{L}_{+}: C_{\eta}([\sigma, \infty) ; E) \rightarrow C_{\eta}([\sigma, \infty) ; F) .
$$

Here, $E=D_{B}(\theta)$ and $F=D_{B}(\theta+1)$, as in $\S 4$.

Proof. We prove the result for $\mathcal{L}_{-}$; the result for $\mathcal{L}_{+}$can be proved similarly. Fix any $f \in C_{\eta}((-\infty, \sigma] ; E)$ and define $u(\cdot)=\int_{-\infty}^{\cdot} \Phi(\cdot, s) f(s) d s$. From (5.2),

$$
u(t)=\int_{-\infty}^{t} e^{(t-s) B} f(s) d s+\int_{-\infty}^{t} \int_{s}^{t} e^{(t-\tau) B} B_{1}(\tau) \Phi(\tau, s) d \tau f(s) d s
$$

The first term is in $C_{\eta}((-\infty, \sigma] ; F)$, by Proposition 4.2 , with

$$
\left\|\int_{-\infty}^{\cdot} e^{(\cdot-s) B} f(s) d s\right\|_{\eta, \sigma,-, F} \leq K_{-}\|f\|_{\eta, \sigma,-, E}
$$

Rewrite the second term as

$$
\int_{-\infty}^{t} e^{(t-s) B} \int_{-\infty}^{\tau} B_{1}(\tau) \Phi(\tau, s) f(s) d s d \tau:=\int_{-\infty}^{t} e^{(t-\tau) B} g(\tau) d \tau
$$

Using Proposition 4.2 again, to complete the proof it will suffice to show that $g \in$ $C((-\infty, \sigma] ; E)$ and that there is a constant $C$, independent of $f$, such that $\|g\|_{\eta, \sigma,-, E} \leq$ $C\|f\|_{\eta, \sigma,-, E}$. We have for all $\tau \leq \sigma$, denoting $m=\max _{0 \leq \tau \leq T}\left\|B_{1}(\tau)\right\|_{L\left(E^{\rho}, E\right)}$,

$$
\begin{aligned}
e^{-\eta \tau}\left|\int_{-\infty}^{\tau} B_{1}(\tau) \Phi(\tau, s) f(s) d s\right|_{E} & \leq m \tilde{M} \int_{-\infty}^{\tau}(\tau-s)^{-\rho} e^{\tilde{\omega}(\tau-s)} e^{-\eta(\tau-s)} e^{-\eta s}|f(s)|_{E} d s \\
& \leq m \tilde{M}\|f\|_{\eta, \sigma,-, E} \cdot \int_{-\infty}^{\tau}(\tau-s)^{-\rho} e^{(\tilde{\omega}-\eta)(\tau-s)} d s
\end{aligned}
$$

Since $\rho<1, \int_{-\infty}^{\tau}(\tau-s)^{-\rho} e^{(\tilde{\omega}-\eta)(\tau-s)} d s<\infty$, and the proposition is proven.

In our problem we will only use $\rho=0$, i.e., $E^{\rho}=E$.

Corollary 5.3 (to Proposition 5.1). For the linear homogeneous periodic problem $(2.8)$, if $\|\alpha\|_{\infty}:=\max _{0 \leq t \leq T}|\alpha(t)|$ is sufficiently small, then the zero solution 
$v \equiv u \equiv 0$ is exponentially asymptotically stable in $Z$, where $Z$ is any of the spaces $\mathbf{X}, \mathcal{D}(B), E$, and $F$.

Proof. Problem (2.8) defines $\Phi(t, s)$, which can be considered as a block diagonal matrix $\left(\Phi_{n}(t, s)\right)_{n=1}^{\infty}$, where $\Phi_{n}(t, s)$ is a $2 \times 2$ principal fundamental matrix for the problem

$$
\left(\begin{array}{c}
\dot{v}_{n} \\
\dot{u}_{n}
\end{array}\right)=\left(\begin{array}{cc}
0 & \kappa_{n} \tan h\left(\kappa_{n} h\right) \\
-\gamma \kappa_{n}^{2}-(g-\alpha(t)) & -\mu \kappa_{n}^{2}
\end{array}\right)\left(\begin{array}{l}
v_{n} \\
u_{n}
\end{array}\right)
$$

It will suffice to show that the characteristic multipliers $\mu_{n, j}, j=1,2, n \geq 1$ of (5.4) (i) go to zero as $n \rightarrow \infty$ for all $\|\alpha\|_{\infty}$ and (ii) have modulus less than 1 for all $n$ if $\|\alpha\|_{\infty}$ is sufficiently small.

Fix an $n$ and denote $c_{n}=\mu \kappa_{n}^{2}, a_{n}=\left(\gamma \kappa_{n}^{2}+g\right) \kappa_{n} \tan h\left(\kappa_{n} h\right)$. Rescale the time variable by $\tau=c_{n} t$ and apply Grönwall's inequality to (5.4) with $\alpha \neq 0$ considered as a perturbation of (5.4) with $\alpha \equiv 0$ to conclude that

$$
\left|\mu_{n}^{ \pm}\right|<\left(-\frac{c_{n}}{2}+\chi_{n}+m \frac{\|\alpha\|_{\infty}}{c_{n}}\right)
$$

where $\chi_{n}:=0$, if $c_{n}^{2} \leq 4 a_{n}$, and $\chi_{n}:=\delta_{n}$, if $c_{n}^{2} \geq 4 a_{n}$, where $\delta_{n}:=\frac{1}{2} \sqrt{c_{n}^{2}-4 a_{n}}$, and $m$ is a constant uniformly bounded in $n$. Results (i) and (ii) follow from easy but tedious asymptotics in (5.5), using $\kappa_{n} \rightarrow \infty$ as $n \rightarrow \infty$.

6. The nonlinearities in equations (2.6)-(2.7). Fix a $\theta \in(0,1)$ and denote $E=D_{B}(\theta), F=D_{B}(\theta+1)$, where $B$ is in equation (2.9). We can write equations (2.6)-(2.7) abstractly as

$$
\frac{d \mathbf{w}}{d t}=\left(B+B_{1}(t)\right) \mathbf{w}+\mathbf{f}(\mathbf{w})
$$

where $\mathbf{f}(\mathbf{w})=\left(f_{1}(\mathbf{w}), f_{2}(\mathbf{w})\right), \mathbf{w}=(v, u)$. The purpose of this section is to show that $\mathbf{f}: F \rightarrow E$ is $C^{\infty}$, using a result of Lin [15].

Recall that

$$
\begin{gathered}
f_{1}(v, u)=w\left|\nabla_{\mathbf{x}} v\right|^{2}-\nabla_{\mathbf{x}} u \cdot \nabla_{\mathbf{x}} v \\
f_{2}(v, u)=M_{2}(v, u)-\left[M_{2}(v, u)\right], \quad[\quad]=\text { mean value over } D \\
M_{2}(v, u)=-\frac{1}{2}\left|\nabla_{\mathbf{x}} u\right|^{2}+\frac{1}{2} w^{2}\left(1+\left|\nabla_{\mathbf{x}} v\right|^{2}\right)
\end{gathered}
$$

and

$$
w=N(v) u=\phi_{z}(\mathbf{x}, v(\mathbf{x}, t), t)
$$

is constructed from the solution $\phi$ of equations (2.1)-(2.3).

THEOREM 6.1 (Lin [15, Thm. 4.3, "fixed $t$ " part of the proof]). Fix any $r>2$. For sufficiently small $|v|_{H^{r}(D)}$, the map $(v, u) \mapsto N(v) u: \tilde{H}^{r}(D) \times \tilde{H}^{r}(D) \rightarrow H^{r}(D)$ exists and is $C^{\infty}$.

The proof consists of mapping the region $\Omega_{v}:=\{(\mathbf{x}, z):-h<z<v(\mathbf{x}), \mathbf{x} \in D\}$ diffeomorphically to $\Omega_{0}:=\left.\Omega_{v}\right|_{v \equiv 0}$ and solving a perturbation of Laplace's equation in $\Omega_{0}$. The diffeomorphism exists because $|v|_{H^{r}(D)}$ is small and $r>2$; the perturbation is small because $|v|_{H^{r}(D)}$ is, so the implicit function theorem can be applied. The 
proof uses a result of Zolesio [29] on multiplication of elements of Sobolev spaces of fractional order.

To show $\mathbf{f}: F \rightarrow E$ is $C^{\infty}$, we use the following theorem.

Theorem 6.2 (daPrato and Grisvard [8, Thm. 2.4]). Suppose $0<\theta<1$, f : $X_{1} \rightarrow X_{2}$ is Fréchet differentiable and $\left.\mathbf{f}\right|_{Y_{1}}: Y_{1} \rightarrow Y_{2}$ is uniformly Lipschitz, where $Y_{i} \hookrightarrow X_{i}$ are continuously imbedded Banach spaces. Then $\mathbf{f}:\left(Y_{1}, X_{1}\right)_{\theta ; \infty} \rightarrow\left(Y_{2}\right.$, $\left.X_{2}\right)_{\theta ; \infty}$ is continuous.

Corollary 6.3. Suppose $0<\theta<1, E=D_{B}(\theta), F=D_{B}(\theta+1)$, $\mathcal{U}_{1}$ is an open neighborhood of $0 \in \mathcal{D}(B)$ and $\mathcal{U}_{2}$ is an open neighborhood of $0 \in \mathcal{D}\left(B^{2}\right)$. If $\mathbf{f}: \mathcal{U}_{1} \subset \mathcal{D}(B) \rightarrow \mathcal{X}$ and $\left.\mathbf{f}\right|_{\mathcal{U}_{2}}: \mathcal{U}_{2} \subset \mathcal{D}\left(B^{2}\right) \rightarrow \mathcal{D}(B)$ is $C^{\infty}$, then so is $\mathbf{f}: \mathcal{V} \subset F \rightarrow E$, for some $\mathcal{V}$ an open neighborhood of $0 \in F$.

Corollary 6.4. Suppose $0<\theta<1, E=D_{B}(\theta), F=D_{B}(\theta+1), \mathbf{X}=X^{\beta} \times$ $X^{\beta-(1 / 2)}, \mathcal{D}(B)=X^{\beta+(1 / 2)} \times X^{\beta+(1 / 2)}, \beta=\frac{r-1}{2}$, and $r>2$. If the region $D$ is a rectangle, then there is an open neighborhood $\mathcal{V}$ of $0 \in F$ such that $\mathbf{f}$ defined by (6.1)(6.2) satisfies $\mathbf{f}: \mathcal{V} \rightarrow E$ is $C^{\infty}$.

Proof. First of all, if $\beta=\frac{r-1}{2}$, then $\mathcal{D}(B) \subset \tilde{H}^{r}(D) \times \tilde{H}^{r}(D)$ and $\mathcal{D}\left(B^{2}\right)=$ $\left.\left(X^{\beta+1} \times X^{\beta+1}\right) \cap\{v, u): \gamma v+\mu u \in H^{\beta+(3 / 2)}\right\} \subset \tilde{H}^{r+1}(D) \times \tilde{H}^{r+1}(D)$. By Theorem 6.1 , for sufficiently small $\delta>0, \mathbf{f}$ maps $\mathcal{U}_{1}=\left\{(v, u) \in \mathcal{D}(B):|v|_{\tilde{H}^{r}(D)}<\delta\right\}$ into $H^{r-1}(D) \times H^{r-1}(D)$, in fact, it maps into $\tilde{H}^{r-1}(D) \times \tilde{H}^{r-1}(D)$ because clearly $\left[f_{2}\right]=0$ and $\left[f_{1}\right]=0$ is noted in Lin $[15, \S 6]$. Again the result on multiplication of Zolesio [29] is used. Likewise, f maps $\mathcal{U}_{2}=\left\{(v, u) \in \mathcal{D}\left(B^{2}\right):|v|_{\tilde{H}^{r+1}(D)}<\delta\right\}$ into $\tilde{H}^{r}(D) \times \tilde{H}^{r}(D)$. The only thing remaining is to check that $\mathbf{f}(v, u)$ satisfies any boundary conditions required to have (i) $\mathbf{f}(v, u) \in X^{\beta} \times X^{\beta-(1 / 2)}=\mathbf{X}$ when $(v, u) \in \mathcal{D}(B)=X^{\beta+(1 / 2)} \times$ $X^{\beta+(1 / 2)}$ and (ii) $\mathbf{f}(v, u) \in X^{\beta+(1 / 2)} \times X^{\beta+(1 / 2)}$, when $(v, u) \in \mathcal{D}\left(B^{2}\right)$.

The only boundary conditions which might need to be satisfied are of the form $\frac{\partial}{\partial \mathbf{n}} \Delta_{N}^{i} f_{j}=0$, integer $i \geq 0, j=1$ or 2 . Recall that $\beta=\frac{r-1}{2}$. For example, if $\frac{5}{2}>r>2$, then $X^{\beta-(1 / 2)}=\tilde{H}^{r-2}(D), X^{\beta}=\tilde{H}^{r-1}(D), X^{\beta+(1 / 2)}=\tilde{H}^{r}(D) \cap\left\{u: \frac{\partial u}{\partial \mathbf{n}}=0\right.$ on $\left.\partial D\right\}$. Another example is if $\frac{7}{2}>r>\frac{5}{2}$, then $X^{\beta-(1 / 2)}=\tilde{H}^{r-2}(D), X^{\beta}=\tilde{H}^{r-1}(D) \cap\{u$ : $\frac{\partial u}{\partial \mathbf{n}}=0$ on $\left.\partial D\right\}$, and $X^{\beta+(1 / 2)}=\tilde{H}^{r}(D) \cap\left\{u: \frac{\partial u}{\partial \mathbf{n}}=0\right.$ on $\left.\partial D\right\}$. Another example to note is that, if $\frac{9}{2}>r>\frac{7}{2}, X^{\beta+(1 / 2)}=\tilde{H}^{r}(D) \cap\left\{u: \frac{\partial u}{\partial \mathbf{n}}=0, \frac{\partial \Delta u}{\partial \mathbf{n}}=0\right.$ on $\left.\partial D\right\}$.

In our problem, $D$ is a rectangle, say $D=\left\{\mathbf{x}=\left(x_{1}, x_{2}\right): 0<x_{j}<\ell_{j}, j=1,2\right\}$ for some $\ell_{1}, \ell_{2}>0$. The eigenfunctions are $\psi_{n}(\mathbf{x})=\cos \left(\omega_{1, n} x_{1}\right) \cos \left(\omega_{2, n} x_{2}\right)$, where $\omega_{1, n}^{2}+\omega_{2, n}^{2}=\kappa_{n}^{2}$. Because of this explicit information that the $\psi_{n}$ 's are even functions of both $x_{1}$ and $x_{2}$ with respect to 0 , we see that for $r>2, u, v, w \in \tilde{H}^{r}(D)$ are even functions in $x_{1}$ and $x_{2}$ and that

$$
\begin{gathered}
f_{1}=w\left|\nabla_{\mathbf{x}} v\right|^{2}-\nabla_{\mathbf{x}} u \cdot \nabla_{\mathbf{x}} v \in \tilde{H}^{r-1}(D), \\
M_{2}=-\frac{1}{2}\left|\nabla_{\mathbf{x}} u\right|^{2}+\frac{1}{2} w^{2}\left(1+\left|\nabla_{\mathbf{x}} v\right|^{2}\right) \in \tilde{H}^{r-1}(D), \\
f_{2}=M_{2}-\left[M_{2}\right]
\end{gathered}
$$

are even functions of $x_{1}$ and $x_{2}$. Again, the result on multiplication of Zolesio [29] is used. This implies that $\frac{\partial}{\partial \mathbf{n}} \Delta^{i} f_{j}=0$ on $\partial D$ whenever it makes sense in terms of a trace of a distribution. It follows that (i) $\mathbf{f}$ satisfies the boundary conditions to be in $\mathbf{X}=X^{\beta} \times X^{\beta-(1 / 2)}$ whenever $(v, u) \in \mathcal{D}(B)=X^{\beta} \times X^{\beta-(1 / 2)}$ and (ii) $\mathbf{f}$ satisfies boundary conditions to be in $\mathcal{D}(B)=X^{\beta+(1 / 2)} \times X^{\beta+(1 / 2)}$ whenever $(v, u) \in \mathcal{D}\left(B^{2}\right)=$ $\left(X^{\beta+1} \times X^{\beta+1}\right) \cap\left\{(v, u): \gamma v+\mu u \in H^{\beta+(3 / 2)}\right\}$.

This completes the proof of Corollary 6.4. 
THEOREM 6.5. For the nonlinear periodic problem (2.6)-(2.7), if $\beta=\frac{r-1}{2}>\frac{1}{2}$, there is a neighborhood $\mathcal{U}$ of $\mathbf{0} \in F=D_{B}(\theta+1) \subset \mathcal{D}(B)=X^{\beta+(1 / 2)} \times X^{\beta+(1 / 2)}$ in which there is existence and uniqueness of solutions, locally forward in time.

We note that if $\beta>1$, i.e., $r>3$, the solutions are classical, i.e., $C^{2}(D) \times C^{2}(D)$, by the Sobolev imbedding theorem. Theorem 6.5 can be proven by a standard use of the contraction mapping theorem, along with Proposition 4.1 and Corollary 6.4.

We also have the following theorem.

THEOREM 6.6 (linearized stability). For the nonlinear periodic problem (2.6)(2.7), if $\beta>\frac{1}{2}$, then $(v, u)=0 \in F$ is exponentially asymptotically stable in the sense of Liapunov, i.e., locally in $F$, if $\|\alpha\|_{\infty}:=\max _{0 \leq t \leq T}|\alpha(t)|$ is sufficiently small.

Theorem 6.6 can be proven in the usual way, using Proposition 4.1, Corollary 5.3, and Corollary 6.4; see, e.g., the proof of daPrato and Lunardi [9, Thm. 2.2]. The restriction "locally in $F$ " is due to the same restriction in Corollary 6.4.

7. A local center-unstable manifold theorem for problem (2.6)-(2.7). As usual, we fix a $\theta \in(0,1)$.

Definition. $A C^{k}$ local center-unstable manifold in $Z$ for a $T$-periodic problem, i.e., periodic with period $T$,

$$
\frac{d \mathbf{w}}{d t}=\left(B+B_{1}(t)\right) \mathbf{w}+\mathbf{f}(\mathbf{w}), \mathbf{w} \in Z
$$

is a set of the form

$$
\mathcal{M}=\left\{\left(t, \xi_{1}+\mathbf{h}\left(t, \xi_{1}\right)\right): \xi_{1} \in \mathcal{U}_{1}(t), t \in \mathbb{R}\right\} \subseteq \mathbb{R} \times Z,
$$

where for all $t, Z=Z_{1}(t) \oplus Z_{2}(t), \mathcal{U}_{1}(t)$ is a neighborhood of $0 \in Z_{1}(t) \mathbf{h}(t, \cdot): \mathcal{U}_{1} \rightarrow$ $Z_{2}(t)$ is $C^{k}, \mathbf{h}(t, 0) \equiv \mathbf{0}, d_{\mathbf{z}} \mathbf{h}(t, 0) \equiv 0$, where d denotes a Fréchet derivative, and $\mathcal{M}$ is invariant forward in time $t$ for (7.1).

We note from the proof of Corollary 5.3 that, for the linear problem (2.8), there are at most finitely many unstable modes, corresponding to characteristic multipliers of modulus greater than 1 , no matter how large $\|\alpha\|_{\infty}:=\max _{0 \leq t \leq T}|\alpha(t)|$. We assume

$\alpha$ is such that (2.8) has a positive finite number $\nu$ of characteristic multipliers

greater than or equal to 1 .

Correspondingly, there is for each $t \in \mathbb{R}$ a finite-rank projection $P_{1}(t)$ on the Hilbert space $\mathcal{X}$; we denote $P_{2}(t)=I-P_{1}(t)$. In an abuse of notation we use $P_{1}(t)$ to denote the restriction of $P_{1}(t)$ to $E=D_{B}(\theta)$ or $F=D_{B}(\theta+1)$; the latter are not Hilbert spaces, but the linear operators $P_{1}(t)$ are still uniformly bounded in $t$, by interpolation.

In fact, one can choose $P_{1}(t)$ so that the evolution family $\left\{\Phi(t, s) P_{1}(s)\right\}$ satisfies

$$
\Phi(t, s) P_{1}(s)=P_{1}(t) \Phi(t, s), \quad t \geq s .
$$

Moreover, because $\operatorname{dim} P_{1}(s) E=\nu<\infty$ for all $s$, the evolution family $\left\{\Phi(t, s) P_{1}(s)\right\}$ can be extended to satisfy

$$
\begin{gathered}
\Phi(t, s) P_{1}(s)=P_{1}(t) \Phi(t, s), \\
\Phi(t, s) P_{1}(s) \Phi(s, \tau) P_{1}(\tau)=\Phi(t, \tau) P_{1}(\tau)
\end{gathered}
$$


for all $t, s, \tau$; in an abuse of notation, we have not bothered to renotate this extended family. Recall that $\nu<\infty$. By hypothesis $(\mathrm{H})$, there exists $M_{1} \geq 1, \omega_{1}>0$ such that

$$
\begin{array}{cc}
\left\|\Phi(t, s) P_{1}(s)\right\|_{L(E, F)} \leq M_{1} e^{-\omega_{1}(t-s)} & \text { for } t \leq s . \\
\left\|\Phi(t, s) P_{1}(s)\right\|_{L(E)} \leq M_{1} e^{-\omega_{1}(t-s)} & \text { for } t \leq s
\end{array}
$$

i.e., backwards in time. In fact, since all of the characteristic multipliers of $\Phi(t+$ $T, T) P_{1}(t)$ are of modulus greater than or equal to 1 , one may choose positive $\omega_{1}$ as close to 0 as desired, although perhaps at the expense of increasing $M_{1}$. Because of estimate (7.2) and the fact that $\operatorname{dim} P_{1}(s) E=\nu<\infty$ for all $s$, one has a backwards maximal regularity result, as in daPrato and Lunardi [9, Thm. 2.4].

Lemma 7.1(corollary to Proposition 5.2). Assume that $B_{1}(\cdot)$ is periodic with period $T, B_{1}(\cdot):[0, T] \rightarrow L\left(E^{\rho}, E\right)$ is Hölder continuous, hypothesis $(\mathrm{H})$ holds, and $\eta$ is chosen so that $\omega_{1}-\eta<0$, where $\omega_{1}$ is as in (7.2). Then for all $\sigma \in \mathbb{R}$, the map

$$
f(\cdot) \mapsto \int_{\sigma}^{\cdot} \Phi(\cdot, s) P_{1}(s) f(s) d s
$$

defines a bounded linear operator

$$
\tilde{\mathcal{L}}_{+}: C_{\eta}((-\infty, \sigma] ; E) \rightarrow C_{\eta}((-\infty, \sigma] ; F)
$$

By hypothesis $(\mathrm{H})$ and the definitions of $P_{1}(t)$ and $P_{2}(t)$, we have that there exists $M_{2} \geq 1, \tilde{\omega}<0$ such that

$$
\left\|\Phi(t, s) P_{2}(s)\right\|_{L(E, F)} \leq M_{2} e^{\tilde{\omega}(t-s)}, \quad \text { for } t \geq s
$$

by Proposition 5.1 .

LEMMA 7.2 (corollary to Proposition 5.2). Assume that $B_{1}(\cdot)$ is periodic with period $T, B_{1}(\cdot):[0, T] \rightarrow L\left(E^{\rho}, E\right)$ is Hölder continuous, hypothesis $(\mathrm{H})$ holds, and $\eta$ is chosen so that $\tilde{\omega}-\eta<0$, where $\tilde{\omega}$ is as in (7.3). Then for all $\sigma \in \mathbb{R}$, the map

$$
f(\cdot) \mapsto \int_{-\infty}^{\cdot} \Phi(\cdot, s) P_{2}(s) f(s) d s
$$

defines a bounded linear operator

$$
\tilde{\mathcal{L}}_{-}: C_{\eta}((-\infty, \sigma] ; E) \rightarrow C_{\eta}((-\infty, \sigma] ; F)
$$

To help in what follows, here are some comparisons of notation in related papers. Chow and Lu's $[6]-\alpha-\eta,-\beta+\eta$, correspond to our $\omega, \tilde{\omega}$, and their $\eta, C_{\eta}\left(\mathbb{R}^{-}, X\right)$ correspond to our $\eta, C_{\eta}((-\infty, \sigma] ; F)$. Chow, Lin, and Lu's [7] $\alpha$ and $-\beta$ correspond to our $\omega_{1}$ and $\tilde{\omega}$, and their $\gamma$ and $E_{\sigma}^{-}(-\gamma, X)$ correspond to our $-\eta$ and $C_{\eta}((-\infty, \sigma] ; F)$. DaPrato and Lunardi's [9, proof of Thm. 3.1] $\omega_{1}$ and $-\mu$ correspond to our $\tilde{\omega}$ and $\eta$. Chow and Lu's [6] condition $\beta+(k-1) \eta>0$ corresponds to our condition $\tilde{\omega}-k \eta<0$. Chow, Lin, and Lu's [7] spectral gap conditions $\alpha<\gamma \leq k \gamma<\beta$ correspond to our conditions $\omega_{1}+\eta<0, \eta<0, \tilde{\omega}-k \eta<0, \tilde{\omega}<0$, and hypothesis $(\mathrm{H})$. DaPrato and Lunardi's spectral gap condition $\lambda_{1}<0 \leq \lambda_{2}$ and $\omega_{1}+\mu<0$ correspond to our conditions $\tilde{\omega}<0$, hypothesis $(\mathrm{H})$, and $\tilde{\omega}-\eta<0$.

TheOREM 7.3. Assume $(\mathrm{H})$. For any integer $k \geq 1$, there exists a local centerunstable manifold $\mathcal{M}$ of dimension $\nu$ for the nonlinear periodic problem (2.6)--(2.7) 
and hence for a model of the Faraday resonance. If we fix $0<\theta<1, r>2$, then $\mathcal{M} \subset \mathbb{R} \times F$, where $F=D_{B}(\theta+1) \subset \mathcal{D}(B)=X^{r / 2} \times X^{r / 2} \subset \tilde{H}^{r}(D) \times \tilde{H}^{r}(D)$. If $r+\theta>3$ then the solutions are classical.

Proof. Fix any $r>2$ and $0<\theta<1$ and define $E=D_{B}(\theta), F=D_{B}(\theta+$ $1)$. Fix any $\sigma \in \mathbb{R}$. Use Lemma 7.2 to define a bounded linear operator $\tilde{\mathcal{L}}_{-}$, and use Lemma 7.1 to define a bounded linear operator $\tilde{\mathcal{L}}_{+}$. The $\eta$ that one chooses is determined by the $\omega_{1}$ in Lemma 7.1 and $\tilde{\omega}$ in Lemma 7.2. One chooses $\eta<0$ such that $\tilde{\omega}-k \eta<0$ and $\omega_{1}-\eta<0$. By Corollary 6.4, there is a neighborhood of $\mathbf{0} \in \mathcal{U} \subset F$ such that $\mathbf{f}: \mathcal{U} \rightarrow E$ is $C^{\infty}$. The proofs of Chow and Lu [6, $\S \S 3$ and 4] for autonomous problems and Chow, Lin, and Lu [7, Lem. 3.1 and 3.2 and Thm. 3.3] for nonautonomous problems work just as well. First, one defines a bounded linear operator $\mathcal{T}: C_{\eta}((-\infty, \sigma] ; E) \rightarrow C_{\eta}((-\infty, \sigma] ; F)$ by $\mathbf{f}(\cdot) \mapsto \int_{\sigma}^{\cdot} \Phi(\cdot, s) P_{1}(s) \mathbf{f}(s) d s+$ $\int_{-\infty}^{\cdot} \Phi(\cdot, s) P_{2}(s) \mathbf{f}(s) d s$. After making the usual cutoff function alteration of $\mathbf{f}$ to get a function $\tilde{\mathbf{f}}$ with sufficiently small Lipschitz constant, the solution of the integral equation

$$
\varphi(t)=\Phi(t, \sigma) \xi_{1}+\int_{\sigma}^{t} \Phi(t, s) P_{1}(s) \tilde{\mathbf{f}}(\varphi(s)) d s+\int_{-\infty}^{t} \Phi(t, s) P_{2}(s) \tilde{\mathbf{f}}(\varphi(s)) d s
$$

is denoted by $\varphi\left(t ; \sigma, \xi_{1}\right)$ for $t \leq \sigma, \xi_{1} \in \mathcal{U}_{1}(\sigma)$. The function $\mathbf{h}$ which gives the integral manifold $\mathcal{M}$ is then defined by

$$
\mathbf{h}\left(\sigma, \xi_{1}\right)=\int_{-\infty}^{\sigma} \Phi(\sigma, s) P_{2}(s) \tilde{\mathbf{f}}\left(\varphi\left(s ; \sigma, \xi_{1}\right)\right) d s=\varphi\left(\sigma ; \sigma, \xi_{1}\right)-\xi_{1} .
$$

See also daPrato and Lunardi [9] for autonomous problems. The conclusion about when the solutions are classical follows from (4.2)-(4.4) and the fact that $F=D_{B}(\theta+$ 1) $=\left\{\mathbf{w} \in D(B): B \mathbf{w} \in D_{B}(\theta)\right\}$.

We note that, as usual, $\mathcal{M}$ may depend on $k$ and the choice of the cutoff function used in the proof. If, in addition, $P_{1}(t+T) \equiv P_{1}(t)$, then $\mathbf{h}\left(t+T, \xi_{1}\right) \equiv \mathbf{h}\left(t, \xi_{1}\right)$ follows from $\Phi(t+T, s+T) \equiv \Phi(t, s)$. So, if the linearization has only decaying modes and simple periodic solutions, then we get a periodic center manifold.

THEOREM 7.4 (exponential attractivity with asymptotic phase). For any integer $k \geq 1$ the local center-unstable manifold $\mathcal{M}$ for problems (2.6)-(2.7) is locally exponentially attractive, i.e., there exists a neighborhood $\mathcal{U}$ of $\mathcal{M}$ such that, if $\mathbf{w}(t)=(v(t), u(t))$ is a solution of (2.6)-(2.7) which exists for $t \in[\sigma, \infty)$ and such that $(t, \mathbf{w}(t)) \in \mathcal{U}$ for $t \in[\sigma, \infty)$, then there exists a solution $\mathbf{w}^{*}(t)$ of $(2.6)-(2.7),(\sigma$, $\left.\mathbf{w}^{*}(\sigma)\right) \in \mathcal{M}$, such that $\left|\mathbf{w}(t)-\mathbf{w}^{*}(t)\right|_{F} \rightarrow 0$ as $t \rightarrow \infty$, exponentially.

Proof. If necessary, by taking $\mathcal{U}$ smaller than in Theorem 7.3 , one can make the Lipschitz constant of $\tilde{\mathbf{f}}$ as small as one needs. The proof is then similar to that of Chow and Lu [6, Thm. 5.1]; again, one uses the integral operators $\tilde{\mathcal{L}}_{ \pm}$.

The hypothesis that $(t, \mathbf{w}(t)) \in \mathcal{U}$ for $\sigma \leq t<\infty$ ensures that the solution of (2.6)-(2.7) also satisfies the problem with a cutoff of $\mathbf{f}$.

8. An example. We mention in passing a result of a sequel in preparation. For a square tank, Silber and Knobloch [23], following experimental work of Simonelli and Gollub [24], studied excitation of the $(3,2)$ and $(2,3)$ spatial modes. We were able to calculate an approximate, local center manifold which reduces the dynamics to that of a periodic system of ordinary differential equations

$$
\left\{\begin{array}{l}
\dot{x}=x q(t, x, y) \\
\dot{y}=y q(t, y, x)
\end{array}\right\}
$$


where $q(t, x, y)=a(t) x^{2}+b(t) y^{2}$. Here, $x, y$ correspond to the center directions for the $(3,2)$ and $(2,3)$ spatial modes.

Acknowledgments. The hospitality of the School of Mathematics of the Georgia Institute of Technology is gratefully acknowledged. It is my pleasure to thank Shui-Nee Chow, Anibal Rodriguez-Bernal, Wenxian Shen, and Mary Silber for their help; in particular, the impetus for this work came out of discussions with Chow and Silber. I also thank an anonymous referee for some helpful suggestions. The referee also made me aware of a paper by Lunardi [17]; it appears that, for my particular applied problem, my work uses simpler spaces than in her paper on fully nonlinear parabolic equations.

\section{REFERENCES}

[1] R. A. Adams, Sobolev Spaces, Academic Press, New York, 1975.

[2] S. B. Angenent, Nonlinear anälytic semiflows, Proc. Roy. Soc. Edinburgh Sect. A, 115 (1990), pp. 91-107.

[3] T. B. BENJAMIN AND F. URSELL, The stability of the plane free surface of a liquid in vertical periodic motion, Proc. Roy. Soc. London Ser. A, 225 (1954), pp. 505-515.

[4] P. L. Butzer And H. Berens, Semigroups of Operators and Approximations, Springer-Verlag, New York, 1967.

[5] S. Chen AND R. TRiggiani, Characterization of domains of fractional powers of certain operators arising in elastic systems, and applications, J. Differential Equations, 88 (1990), pp. 279-293.

[6] S.-N. CHOw AND K. LU, Invariant manifolds for flows in Banach spaces, J. Differential Equations, 74 (1988), pp. 285-317.

[7] S.-N. CHOw, X.-B. LIN, AND K. LU, Smooth invariant foliations in infinite-dimensional spaces, J. Differential Equations, 94 (1991), pp. 266-291.

[8] G. DAPRATO AND P. GRISVARD, Equations d'evolution abstraites nonlineaires de type parabolique, Ann. Mat. Pura Appl. (4), 120 (1979), pp. 329-396.

[9] G. DAPRATO AND A. LUNARDI, Stability, instability, and center manifold theorem for fully nonlinear autonomous parabolic equations in Banach space, Arch. Rational Mech. Anal., 101 (1988), pp. 115-141.

[10] S. DouAdY, Experimental study of the Faraday instability, J. Fluid Mech., 221 (1990), pp. 383-409.

[11] P. GRISVARD, Caracterisation de quelques espaces d'interpolation, Arch. Rational Mech. Anal., 25 (1967), pp. 40-63.

[12] D. HENRY, Geometric theory of semilinear parabolic equations, in Lecture Notes in Mathematics, vol. 840, Springer-Verlag, Berlin, 1981.

[13] P. Holmes, Chaotic motions in a weakly nonlinear model for surface waves, J. Fluid Mech., 162 (1986), pp. 365-388.

[14] X. M. Gu And P. R. Sethna, Resonant surface waves and chaotic phenomena, J. Fluid Mech., 183 (1987), pp. 543-565.

[15] X.-B. LIN, Existence theory for damped gravity waves in a closed rectangular basin, Arch. Rational Mech. Anal., 114 (1991), pp. 267-295.

[16] J. L. Lions AND E. MAGEnes, Nonhomogeneous Boundary Value Problems and Applications, vol. I, Springer-Verlag, New York, 1972.

[17] A. LunARDI, Stability of the periodic solutions to fully nonlinear parabolic equations in Banach spaces, Differential Integral Equations, 1 (1988), pp. 253-279.

[18] J. Miles And D. Henderson, Parametrically forced surface waves, Ann. Rev. Fluid Mech., 22 (1990), pp. 143-165.

[19] S. M. NIKOL'SKII, On imbedding, continuation, and approximation theorems for differentiable functions of several variables, Uspekhi Mat. Nauk, 16 (1961), pp. 63-114 (in Russian); English translation, Russian Math. Surveys, 16 (1961), pp. 55-104.

[20] - - Approximation of Functions of Several Variables and Imbedding Theorems, SpringerVerlag, Berlin, 1975. 
[21] A. PAZY, Semigroups of Linear Operators and Applications to Partial Differential Equations, Springer-Verlag, New York, 1983.

[22] A. Rodriguez-BERNAL, On the generation of analytic semigroups by a class of damped wave equations, to appear.

[23] M. Silber AND E. KNOBloch, Parametrically excited surface waves in square geometry, Phys. Letters A, 137 (1989), pp. 349-354.

[24] F. Simonelli AND J. P. Gollub, Surface wave mode interactions: Effects of symmetry and degeneracy, J. Fluid Mech., 199 (1989), pp. 471-494.

[25] E. SinESTRARI, On the abstract Cauchy problem of parabolic type in spaces of continuous functions, J. Math. Anal. Appl., 107 (1985), pp. 16-66.

[26] L. N. SLOBodeCKII, Generalized Sobolev spaces and their application to boundary value problems for partial differential equations, Leningradskiŭ Gosudarstvennyı̌ Pedagogičeskĭ Institut im A. I. Gercena Učenye Zapiski, 197 (1958), pp. 54-112 (in Russian); English translation, Amer. Math. Soc. Transl. Ser. 2, 57 (1966), pp. 207-275.

[27] L. TURYN, The damped Mathieu equation, Quart. Appl. Math., 51 (1993), pp. 389-398.

[28] S. A. VAN Gils AND A. VANDERBAuwhede, Center manifolds and contractions on a scale of Banach spaces, J. Funct. Anal., 72 (1987), pp. 209-224.

[29] J. L. Zolesio, Multiplication dans les espaces de Besov, Proc. Roy. Soc. Edinburgh Sect. A, 78 (1977), pp. 113-117. 